The Astrophysical Journal, 281 : 141-147, 1984 June 1

(C) 1984. The American Astronomical Society. All rights reserved. Printed in U.S.A

\title{
THE CLUSTERS OF M33
}

\author{
J. G. COHEN \\ Palomar Observatory, California Institute of Technology \\ AND \\ S. E. Persson and L. Searle \\ Mount Wilson and Las Campanas Observatories \\ Received 1983 June 17; accepted 1983 December 1
}

\begin{abstract}
A selection of clusters in M33 is studied using three different observational techniques which give consistent quantitative results. M33 contains clusters which resemble galactic globular clusters as well as clusters as young as $10^{8}$ years; the luminous clusters thus have an age range similar to those of the Magellanic Clouds rather than those of our Galaxy or M31. The four true globular clusters in our sample are all more metal poor than 47 Tuc. Based on limited data, the carbon star contribution to the integrated infrared light of the intermediate age clusters in M33 is approximately the same as in the intermediate age clusters in the Magellanic Clouds.

Subject headings: clusters: globular — clusters: open — galaxies: individual — stars: abundances stars: evolution
\end{abstract}

\section{INTRODUCTION}

Our understanding of the integrated light of star clusters is sufficiently advanced that quantitative estimates of age and metallicity can be made by means of several different techniques. Three of these have been tested on nearby systems, such as the galactic globular clusters and the clusters of the Magellanic Clouds. These are analysis of moderate resolution optical spectroscopy (see Cohen 1982 and earlier references therein; Rabin 1982), optical spectrophotometry (see Searle and Zinn 1978; Zinn 1980; Searle, Wilkinson, and Bagnuolo 1980), and broad-band infrared photometry (Persson et al. 1983; Frogel, Cohen, and Persson 1983) which has been applied as well to the cluster system of M31 (Frogel, Persson, and Cohen 1980, hereafter FPC 80). In this paper we apply these three techniques to a study of the clusters of the nearby late-type spiral M33. The clusters of this galaxy have been surveyed by Hiltner (1960), Melnick and D'Odorico (1978), and recently by Christian and Schommer (1982). M33, unlike our Galaxy or M31, is a late-type spiral [classified as Sc(s) II-III by Sandage and Tammann 1981]. It contains numerous early-type and $\mathbf{M}$ supergiants (see, for example, Humphreys and Sandage 1980) and gives ample evidence of strong current star formation. Since M33 is almost face-on, unlike M31, a magnitude-limited survey of the cluster population at visual wavelengths is bound to find mostly younger clusters (which are more luminous for a fixed total mass of stars) if they are present. We therefore brought to bear the three techniques listed above on the cluster system of M33 with the aim of throwing some light on the history of star formation and heavy element formation in this galaxy. Consistent results were obtained from analyzing the three independent data sets as described in $\S \S$ II-IV. We find that the cluster system of M33 contains a heterogenous collection of objects that spans the full range of age; it contains clusters resembling galactic globulars as well as clusters as young as $10^{8}$ years. Possible similarities between the star formation history of our Galaxy and M31, as compared to M33 and the Magellanic Clouds, are indicated in $\S \mathrm{V}$.
II. OBSERVATIONAL TECHNIQUES

a) Spectra

Clusters were selected from Hiltner (1960) to cover the full range in $B-V$ color. They were observed during 1981 August and 1982 July using the Double Spectrograph (Oke and Gunn 1982) at the Cassegrain focus of the $5 \mathrm{~m}$ telescope at Palomar Observatory. In the first run the detector on the blue camera was an RCA CCD chip as described in Oke and Gunn (1982); it was used with a 5" diameter aperture. In 1982 July the detector was a new two-dimensional version of the intensified reticon array designed by $\mathrm{S}$. Shectman; it was used with a slit $2^{\prime \prime}$ wide. This setup gave spectra with a higher signal-to-noise ratio at very low signal levels (due to the readout noise of the RCA chip). In both cases the spectra are fully sky subtracted. A $5^{\prime \prime}$ diameter aperture is large enough to contain more than $70 \%$ of the total luminosity of each of the M33 clusters as can be seen from comparison of our fluxes at $5500 \AA$ with Hiltner's visual photometry through an aperture with a diameter of $12^{\prime \prime}$. In 1982 April and June five galactic globular clusters were observed with the same equipment as for the 1981 August data, but using an aperture whose diameter was $10^{\prime \prime}$. The aperture was trailed slightly over the core of each of the globular clusters in an effort to obtain a more representative sample of the integrated light. The grating used in 1981 August produces $8 \AA$ per $30 \mu \mathrm{m}$ pixel at the focal plane, while the 600 line $\mathrm{mm}^{-1}$ grating used in 1982 July gave $1.5 \AA$ per $30 \mu \mathrm{m}$ in the blue camera. Approximate equivalent widths using a continuum extrapolated from the wavelength intervals $3905-3915 \AA$ and 4020-4060 $\AA$ were measured for the Ca II feature at $3933 \AA$. The approximate equivalent widths of the Balmer lines $\mathrm{H} \delta$, $\mathrm{H} \beta$, and $\mathrm{H} \gamma$ were also measured. The data are listed in Table 1 , where the number of individual spectra (and the detector used) for each cluster are indicated.

\section{b) Spectrophotometry of the M33 Clusters}

In order to compare the clusters in M33 with those of the Clouds and with those of the Galaxy, spectrophotometric data 
TABLE 1

Equivalent Widths For M33 Clusters

\begin{tabular}{|c|c|c|c|c|}
\hline Object & No. of Obs. ${ }^{a}$ & $\begin{array}{c}W_{\lambda}(3933) \\
(\AA)\end{array}$ & $\underset{(\AA)}{\operatorname{Median} \mathrm{H} \beta, \gamma, \delta}$ & $B-V^{\mathrm{b}}$ \\
\hline $\mathrm{a}$ & $2 \mathrm{C}+1 \mathrm{~S}$ & $2.8 \pm 1$ & $7.1 \pm 0.5$ & 0.21 \\
\hline$b$ & $1 C+1 S$ & $2.3 \pm 1$ & $8.4 \pm 0.7$ & 0.40 \\
\hline $\mathrm{d} \ldots \ldots \ldots$ & $1 C+1 S$ & $3.2 \pm 1.5$ & $6.7 \pm 0.5$ & 0.21 \\
\hline e............ & $1 \mathrm{C}+1 \mathrm{~S}$ & $6.7 \pm 1$ & $3.3 \pm 0.6$ & 0.77 \\
\hline $\mathrm{f} \ldots$ & $1 \mathrm{C}$ & $\leq 5.0$ & 5.7 & 0.58 \\
\hline $\mathrm{g} \ldots \ldots \ldots$ & $1 C+1 S$ & $2.6 \pm 1$ & $9.3 \pm 0.3$ & 0.28 \\
\hline$h \ldots \ldots \ldots$ & 1S & $8 . \overline{9}$ & 2.7 & 0.75 \\
\hline $\mathrm{i} \ldots \ldots \ldots$ & $1 \mathrm{C}+1 \mathrm{~S}$ & $2.2 \pm 1$ & $7.0 \pm 0.5$ & 0.17 \\
\hline $\mathrm{m} \ldots \ldots \ldots$ & $1 \mathrm{C}+1 \mathrm{~S}$ & $9.9 \pm 1$ & $\leq 3.5$ & 0.80 \\
\hline$q \ldots \ldots \ldots$ & $1 \mathrm{C}$ & $\leq \overline{6.1}$ & 3.5 & 0.74 \\
\hline $\mathrm{w} \ldots \ldots \ldots$ & $1 \mathrm{C}$ & $\leq 4.7$ & 8.8 & 0.23 \\
\hline
\end{tabular}

${ }^{1} \mathrm{C}$ denotes the RCA CCD detector; $\mathrm{S}$ denotes the two-dimensional photon counting detector of Shectman (see text).

b $B-V$ colors from Hiltner 1960.

were obtained, and from them, colors on the Thuan and Gunn (1976) system were synthesized. Spectral flux distributions for nine of the clusters in M33 were measured in 1979 using the multichannel spectrometer of the Hale reflector. In the same observing run 12 of the standard stars that Zinn (1980) adopted for his program of $u v g r$ photometry of galactic globular clusters were also observed.

Absolute flux densities in 48 overlapping wavelength bands were derived using observations of standard stars of Oke and Schild (1970) made on the same nights and standard calibration procedures. The bandwidth was $160 \AA$ for bands centered on $\lambda 3220$ (80) $\lambda 5800 \AA$ and $360 \AA$ for the remaining bands centered on $\lambda 5780$ (180) $\lambda 8400 \AA$. These spectral energy distributions were folded with the response functions of the $u, v, g$, and $r$ filters to produce pseudocounts. The response function of each filter is the product of its filter transmission function, the atmospheric transmission function corresponding to one air mass, and the quantum efficiency of an S20 photocathode. The pseudocounts were converted to instrumental magnitudes; the zero points being established, following Thuan and Gunn (1976), from the known energy distribution of the fundamental spectrophotometric standard BD $+17^{\circ} 4708$ whose $g$ magnitude and colors are defined to be 9.500 and 0.000 , respectively. The scale of the transformation was checked by plotting Zinn's colors for the standards listed in Table 2 against the instrumental colors; fitting a line through the origin gives:

$$
\begin{aligned}
& u-g=1.029(u-g)^{\prime} \pm 0.02 \\
& v-g=1.035(v-g)^{\prime} \pm 0.01 \\
& g-r=1.009(g-r)^{\prime} \pm 0.01
\end{aligned}
$$

where the primed values are the instrumental colors. These relations were used to transform all instrumental colors to Zinn's system. Table 2 presents the colors derived from the multichannel scans for 12 of Zinn's standards together with the colors that Zinn adopted for them. The difference in $v-g$ and $g-r$ measured by Zinn and synthesized from the scans never exceeds $0.03 \mathrm{mag}$. This is also the value of the standard deviation of the absolute value of the differences in the $u-g$ color. Table 2 also contains the $u-g, v-g$, and $g-r$ colors for the nine clusters in $\mathrm{M} 33$, as well as the derived reddeningindependent quantities $Q(u g r)$ and $Q(v g r)$, the analogs of Johnson's $Z$ in this photometric system. The definition of these quantities and a demonstration that they are, in fact, reddening
TABLE 2

\begin{tabular}{|c|c|c|c|c|c|c|}
\hline Cluster & $u-g$ & $v-g$ & $g-r$ & $Q(u g r)$ & $Q($ vgr $)$ & Type \\
\hline a ... & -0.04 & -0.21 & -0.18 & 0.15 & -0.09 & II-III \\
\hline b... & 0.44 & 0.04 & -0.04 & 0.48 & 0.06 & IV \\
\hline c ... & -0.13 & -0.28 & -0.27 & 0.17 & -0.10 & II-III \\
\hline d ... & 0.00 & -0.19 & -0.18 & 0.19 & -0.07 & III \\
\hline $\mathrm{e} \ldots .$. & 0.52 & 0.32 & 0.33 & 0.16 & 0.09 & VII \\
\hline$\ldots \ldots \ldots$ & 0.62 & 0.26 & 0.17 & 0.44 & 0.15 & V \\
\hline$h \ldots$ & 0.64 & 0.45 & 0.41 & 0.20 & 0.17 & VI \\
\hline $\mathrm{m} \ldots \ldots \ldots \ldots \ldots \ldots$ & 0.54 & 0.40 & 0.34 & 0.17 & 0.17 & VI \\
\hline $\mathrm{q} \ldots \ldots \ldots \ldots \ldots \ldots$ & 0.34 & 0.25 & 0.29 & 0.02 & 0.05 & VII \\
\hline
\end{tabular}

M33 Cluster and STANDARd STAR Synthesized Colors

A. Cluster Synthesized Colors

\begin{tabular}{|c|c|c|c|c|c|c|}
\hline \multirow[b]{2}{*}{ STANDARD } & \multicolumn{3}{|c|}{ COLORS FROM SCANS } & \multicolumn{3}{|c|}{ COLORS FROM ZINN } \\
\hline & $u-g$ & $v-g$ & $g-r$ & $u-g$ & $v-g$ & $g-r$ \\
\hline SA 94305 & 2.30 & 1.51 & 0.91 & 2.30 & 1.50 & 0.93 \\
\hline $\begin{array}{llll}\text { SA } & 94 & \text { SUS } \\
\text { SA } 95 \quad 134\end{array}$ & 0.24 & 0.06 & -0.02 & 0.30 & 0.06 & -0.05 \\
\hline $\mathrm{BD}+02^{\circ} 3375$ & -0.08 & -0.02 & 0.03 & -0.05 & 0.00 & 0.03 \\
\hline $\mathrm{BD}+21^{\circ} 607 \ldots$ & -0.06 & -0.02 & 0.00 & -0.03 & 0.00 & -0.01 \\
\hline $\mathrm{BD}+28^{\circ} 4211$. & -1.54 & -0.83 & -0.87 & -1.62 & -0.80 & -0.87 \\
\hline SA $113276 \ldots$ & 0.51 & 0.37 & 0.12 & 0.49 & 0.36 & 0.12 \\
\hline SA $113274 \ldots$ & 0.25 & 0.15 & -0.03 & 0.24 & 0.14 & -0.04 \\
\hline SA 92336 . & 1.34 & 0.90 & 0.46 & 1.32 & 0.90 & 0.45 \\
\hline SA $115349^{\circ}$ & 1.44 & 0.97 & 0.53 & 1.42 & 0.97 & 0.51 \\
\hline HD 186293 & 1.57 & 1.07 & 0.60 & 1.60 & 1.08 & 0.59 \\
\hline HD 171164 & 1.57 & 1.04 & 0.55 & 1.59 & 1.06 & 0.54 \\
\hline HD $186312 \ldots \ldots \ldots$ & 2.53 & 1.62 & 0.96 & 2.52 & 1.62 & 0.96 \\
\hline
\end{tabular}

B. Standard Star Synthesized Colors

independent is given by Zinn (1980). In using these quantities for M33 clusters we are assuming, of course, that the reddening law is the same in M33 and in the Galaxy.

\section{c) Broad-Band Optical and Infrared Photometry}

Broad-band $(J H K)$ infrared photometry was obtained for eight clusters in M33 on the Hale $5 \mathrm{~m}$ reflector in 1981 September. Standard infrared chopping and beam-switching techniques were used with an aperture $5^{\prime \prime}$ in diameter. Optical photometric data were obtained simultaneously with a GaAs tube and Gunn $r$ filter (Thuan and Gunn 1976). During alternate chops of the telescope secondary mirror, when the infrared detector was sampling the "reference beam" position, the cluster light was directed to the optical photometer. The infrared and optical light was thus measured in exactly the same way for the cluster and both reference beams, which were separated from the signal beams by a fixed $25^{\prime \prime}$. All three beam locations were checked for contaminating stars and irregular background galaxy light. Although an irregular background (of a markedly different color from that of the cluster) could introduce errors into the magnitudes, the clusters are, in all cases, much brighter than the background in a $5^{\prime \prime}$ aperture, and the effects upon the cluster colors should be small.

The sizes, profiles, and locations of the infrared and optical apertures were carefully compared and found to be nearly indistinguishable. The resulting magnitudes, on the systems of Elias et al. (1982) and Thuan and Gunn (1976), are given in Table 3. (Note that a $5^{\prime \prime}$ aperture does not in general include all the cluster light.) To check the quality of the $r-H$ and infrared colors, we also measured six clusters in M31 during the same observing run. The $J-K$ and $H-K$ colors are in reasonable agreement with those in Frogel, Persson, and Cohen (1980), the mean differences being $-0.01 \pm 0.03(1 \sigma)$ mag for 
TABLE 3

M33 ANd M31 Cluster Photometry

\begin{tabular}{|c|c|c|c|c|c|c|c|c|}
\hline Cluster & $r$ & $J$ & $H$ & $K$ & $(J-K)_{0}$ & $(H-K)_{0}$ & $(V-H)_{0}$ & $(V-H)_{0}($ FPC 80$)$ \\
\hline M33a . & $16.76(2)$ & $15.49(4)$ & $15.08(4)$ & $14.89(7)$ & $0.58(8)$ & $0.18(8)$ & $1.56(6)$ & . \\
\hline M33b ......... & $17.43(2)$ & $15.85(4)$ & $15.20(3)$ & $14.84(11)$ & $0.99(11)$ & $0.35(11)$ & $2.34(6)$ & $\ldots$ \\
\hline M33e ......... & $16.32(2)$ & $14.64(4)$ & 14.11(3) & $13.96(4)$ & $0.66(6)$ & $0.14(5)$ & $2.31(6)$ & $\ldots$ \\
\hline M33g ......... & $18.03(2)$ & $16.74(4)$ & $16.20(4)$ & $16.03(9)$ & $0.69(10)$ & $0.16(10)$ & $1.77(6)$ & $\ldots$ \\
\hline M $33 \mathrm{~h} \ldots \ldots \ldots$ & $17.19(2)$ & $15.48(4)$ & $14.96(3)$ & $14.88(5)$ & $0.58(6)$ & $0.07(6)$ & $2.34(6)$ & $\ldots$ \\
\hline M33i $\ldots \ldots \ldots$ & $17.89(2)$ & $16.44(5)$ & $15.92(4)$ & $15.63(7)$ & $0.79(9)$ & $0.28(8)$ & $1.97(6)$ & . \\
\hline M33s .. & $18.30(2)$ & $16.53(4)$ & $15.96(4)$ & $15.75(7)$ & $0.76(8)$ & $0.20(8)$ & $2.49(6)$ & $\ldots$ \\
\hline M33q ......... & $17.20(2)$ & & $14.96(3)$ & $14.84(5)$ & & $0.12(6)$ & $2.35(6)$ & \\
\hline M $31-23 \ldots \ldots \ldots$ & $14.95(3)$ & $13.26(4)$ & $12.69(3)$ & $12.51(4)$ & $0.70(6)$ & $0.16(5)$ & $2.20(6)$ & 2.23 \\
\hline M31-42 ....... & $14.02(2)$ & $11.82(4)$ & $11.14(3)$ & $10.92(3)$ & $0.75(5)$ & $0.17(4)$ & $2.52(6)$ & 2.53 \\
\hline M31-44 ....... & $14.86(2)$ & $12.94(4)$ & $12.31(3)$ & $12.14(3)$ & $0.72(5)$ & $0.14(4)$ & $2.43(6)$ & 2.40 \\
\hline M31-112..... & $15.88(2)$ & $14.33(4)$ & $13.85(3)$ & $13.70(3)$ & $0.58(5)$ & $0.13(4)$ & $1.88(6)$ & 1.89 \\
\hline M31-118...... & $15.76(2)$ & $14.02(4)$ & $13.46(3)$ & $13.27(3)$ & $0.70(5)$ & $0.17(4)$ & $2.26(6)$ & 2.25 \\
\hline M31-282 ..... & $14.03(2)$ & $12.03(4)$ & $11.39(3)$ & 11.19(3) & $0.79(5)$ & $0.18(4)$ & $2.73(6)$ & 2.71 \\
\hline
\end{tabular}

NOTE.-Observational $1 \sigma$ uncertainties in hundredths of a magnitude are given in parentheses after each entry.

$J-K$ and $+0.03 \pm 0.03(1 \sigma)$ mag for $H-K$. The correlation of the newly measured $r-H$ colors with the $V-H$ colors in Frogel, Persson, and Cohen (1980) is very tight, with a least squares fit giving $V-H=1.407(r-H)-0.721$. Although different from that found for elliptical galaxies (Persson et al. 1980), this equation gives similar results over the narrow range of color considered here. Application of this transformation gives $V-H$ colors for all the M31 and M33 clusters. To correct for reddening a value $E(B-V)=0.03$ for M33 (McClure and Racine 1969) was assumed. The last column of Table 3 lists the reddening corrected $(V-H)_{0}$ colors, with the new (transformed) and old (Frogel, Persson, and Cohen 1980) colors for M31 listed to show the quality of the fit.

\section{d) Velocities}

The spectra of eight of the M33 clusters in our sample are of high enough precision and resolution to be used to measure radial velocities. These measurements are listed in Table 4. For two of the clusters, radial velocities have been measured from more than one spectrum. Because of the lack of suitable templates, these velocities were determined by measuring the central wavelength of the $\mathrm{Ca}$ II line at $3933 \AA$ and the Balmer lines from $\mathrm{H} 11$ to $\mathrm{H} \beta$, excluding $\mathrm{H} \epsilon$. The errors listed in Table 4 reflect the dispersion of the individual lines about the tabulated mean heliocentric velocity for each cluster. For the three clusters with accurately determined radial velocities, in two of the three cases these values agree to within $20 \mathrm{~km} \mathrm{~s}^{-1}$ with that of $\mathrm{H}$ I in the disk of M33 as studied by Rogstad, Wright, and Lockhart (1976). The discrepant case is M33 cluster e, which is

TABLE 4

Heliocentric Radial Velocities of M33 Clusters

\begin{tabular}{lcccc}
\hline \hline Cluster & No. of Obs. & $\begin{array}{c}V_{r} \\
\left(\mathrm{~km} \mathrm{~s}^{-1}\right)\end{array}$ & $\begin{array}{c}\text { Error } \\
\left(\mathrm{km} \mathrm{s}^{-1}\right)\end{array}$ & $\begin{array}{c}V_{r}(\mathrm{H} \mathrm{I})^{\mathrm{a}} \\
\left(\mathrm{km} \mathrm{s}^{-1}\right)\end{array}$ \\
\hline $\mathrm{a} \ldots \ldots \ldots$ & 3 & -245 & 30 & -235 \\
$\mathrm{~b} \ldots \ldots \ldots \ldots$ & 1 & -105 & 100 & -235 \\
$\mathrm{~d} \ldots \ldots \ldots \ldots$ & 1 & -255 & 30 & -235 \\
$\mathrm{e} \ldots \ldots \ldots \ldots$ & 2 & -125 & 30 & -225 \\
$\mathrm{~g} \ldots \ldots \ldots \ldots$ & 1 & -75 & 70 & -135 \\
$\mathrm{~h} \ldots \ldots \ldots$ & 1 & -100 & 100 & -120 \\
$\mathrm{i} \ldots \ldots \ldots \ldots$ & 1 & -20 & 70 & -110 \\
$\mathrm{~m} \ldots \ldots \ldots$ & 1 & -220 & 100 & -245 \\
\hline
\end{tabular}

${ }^{a}$ From Rogstad, Wright, and Lockhart 1976. classified spectroscopically and photometrically (in $\S \S$ III and IV) as a globular cluster and therefore is not likely to be confined to the disk of M33. This is in agreement with the conclusions of Christian and Schommer (1983). Of the four clusters in common, the radial velocities of three agree to within the errors of our measurements.

\section{THE AGE RANGE OF THE M33 CLUSTERS}

Figure 1 shows the $\mathrm{Ca}$ II absorption-line strengths versus the median strength of $\mathrm{H} \delta, \mathrm{H} \gamma$, and $\mathrm{H} \beta$ as measured from our moderate dispersion spectra for 11 M33 clusters and for five galactic globular clusters. The locus of the galactic globular clusters is shown; the extension toward stronger $\mathrm{Ca}$ II lines is a consequence of the addition of globulars more metal rich than the five that we observed. Rabin (1982) has discussed the interpretation of the equivalent of Figure 1 as applied to observations of the integrated spectra of clusters in the Magellanic Clouds. The positions occupied by the bluer M33 clusters in our sample (whose spectra are dominated in the blue by the Balmer lines) correspond to those of Magellanic Cloud clusters

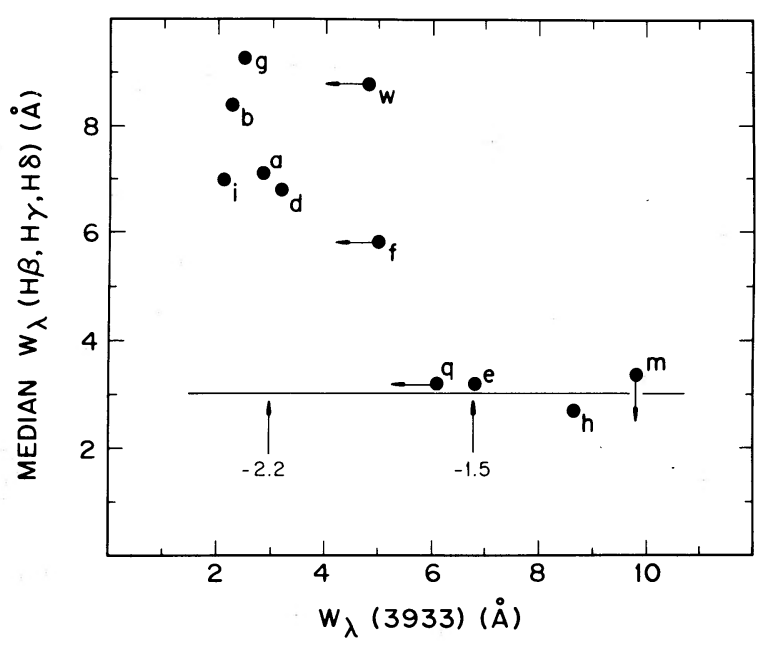

Fig. 1.-The median equivalent width of the three Balmer lines $\mathrm{H} \beta, \mathrm{H} \gamma$, and $\mathrm{H} \delta$ is plotted as a function of the equivalent width of the $\mathrm{Ca}$ Il line at 3933 $\AA$ for $11 \mathrm{M} 33$ clusters. The locus of galactic globular clusters is indicated by the solid line. The location of globular clusters along this locus with $[\mathrm{Fe} / \mathrm{H}]=$ -2.2 and -1.5 is indicated. 
of class III-V using the photometric classification scheme of Searle, Wilkinson, and Bagnuolo (1980, hereafter SWB), or of ages less than 1-6 billion years. From Figure 1 we infer that M33 clusters e, h, q, and $\mathrm{m}$ are the only objects among the sample of 11 clusters whose spectra are similar to those of galactic globular clusters of any metallicity. They are also the only clusters among the sample with $B-V$ colors greater than $0.6 \mathrm{mag}$.

The range in age of the M33 clusters from relatively young to $13 \times 10^{9}$ years inferred from the optical spectra is confirmed by the spectrophotometry of nine clusters. In Figure 2 the M33 clusters are plotted in the $Q(u g r), Q(v g r)$ plane. The physical interpretation of this $Q-Q$ array is discussed by SWB. In Figure $2 a$ the clusters are plotted together with a boundary that encloses the globular clusters of the Galaxy. This enclosed region is divided into two zones. All galactic globular clusters that are further than $9 \mathrm{kpc}$ from the Galactic center fall in Zone A, while those that fall in Zone B are closer to the center than 9 $\mathrm{kpc}$ and also lie within $4 \mathrm{kpc}$ of the Galactic plane. The location of an age-sequence of clusters that have a solar metalcontent is also plotted in Figure $2 a$, with ages in $10^{9}$ years marked along it. This sequence is based on models by Bagnuolo (1976) and is taken from Figure 1 of SWB.

Only four of the M33 clusters (Hiltner's clusters q, e, m, and h) fall within the region occupied by the Galaxy's globular cluster, and all of these fall in Zone A. These are the same four clusters identified spectroscopically as globulars. It is evident from Figure 2 and from the discussion in SWB that the other five M33 clusters in the sample with spectrophotometry are younger and more metal rich than the clusters $\mathrm{q}, \mathrm{e}, \mathrm{m}$, and $\mathrm{h}$.

In Figure $2 b$ the data points for the M33 clusters are superposed on a diagram showing the location in the $Q-Q$ plane of clusters in the LMC. SWB arbitrarily divided this region into zones that served to define the SWB classification of Cloud clusters, and these zones are indicated in Figure 2. The clusters of M33 occupy roughly the same territory as the LMC clusters and we can therefore assign SWB types to them; these are listed in Table 2.

The three M33 clusters with negative values of $Q(v g r)$ are
Hiltner's clusters a, c, and d. They fall near the boundary of the type II and type III zones. Rabin (1982) has assigned an age of roughly $10^{8}$ years to this boundary. The LMC clusters NGC 1854 and NGC 2136 lie close to clusters a, c, and $\mathrm{d}$ in the $Q-Q$ diagram and presumably have roughly comparable age. On the basis of color-magnitude arrays, these two LMC clusters are assigned ages of $3 \times 10^{7}$ and $4 \times 10^{7}$ years, respectively, in Hodge's (1981) compilation. Approximate ages for the youngest M33 clusters can be deduced assuming the spiral pattern moves through the gaseous disk at a rate given by density wave theory, while the clusters move only with the appropriate rotation rate for their distance from the nucleus of the galaxy. Such calculations have been made for O stars in M33 by Kaufman (1981), for example, using the $21 \mathrm{~cm}$ rotation curve of Rogstad, Wright, and Lockhart (1976). Using these kinematic data and the distance modulus of Sandage and Carlson (1982), we obtain ages of $5 \pm 1 \times 10^{7}$ years, where the uncertainty includes only that of the distance from the cluster to the nearest spiral arm projected onto the plane of the sky. In any case clusters a, c, and d are certainly young and were formed within the last $1 \%$ of M33's lifetime. Integrated colors give little information concerning the chemical composition of such young clusters.

The two M33 clusters with $Q(u g r) \sim 0.4$ are Hiltner's b and f, and they have SWB types IV and V, respectively. In its photometric properties, cluster $b$ is a fairly close match with NGC 2107 in the Large Cloud and with NGC 269 in the Small Cloud. According to the calibration shown in Figure $2 a$, these clusters have ages of roughly $10^{9}$ years. This is consistent with Aaronson and Mould's (1982) upper limit of $1.5 \times 10^{9}$ years to the age of NGC 2107. Cluster $\mathrm{f}$ has photometric properties which rather closely match the LMC clusters NGC 1846 and NGC 1987. Rabin's (1982) spectra of these two LMC clusters show NGC 1987 to be comparable in age to NGC 2209, a cluster Rabin estimates to have an age of $2 \times 10^{9}$ years. NGC 1846 is somewhat older as is evidenced by its weaker Balmer lines (Rabin's data concerning this point are corroborated by Danziger's [1973] earlier observations), and an age of $4 \times 10^{9}$ years for NGC 1846 is our best estimate. We conclude that

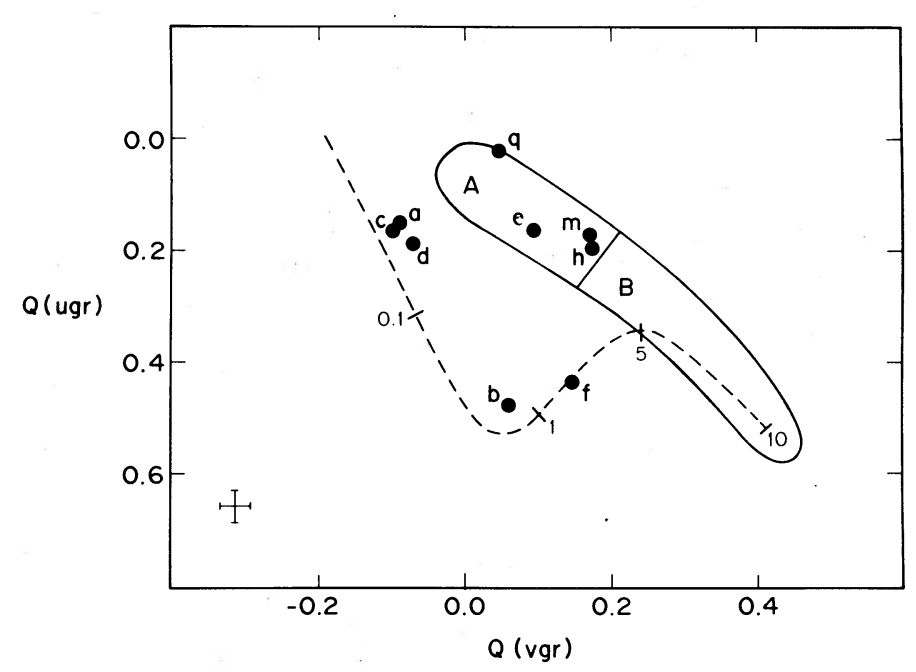

FIG. $2 a$

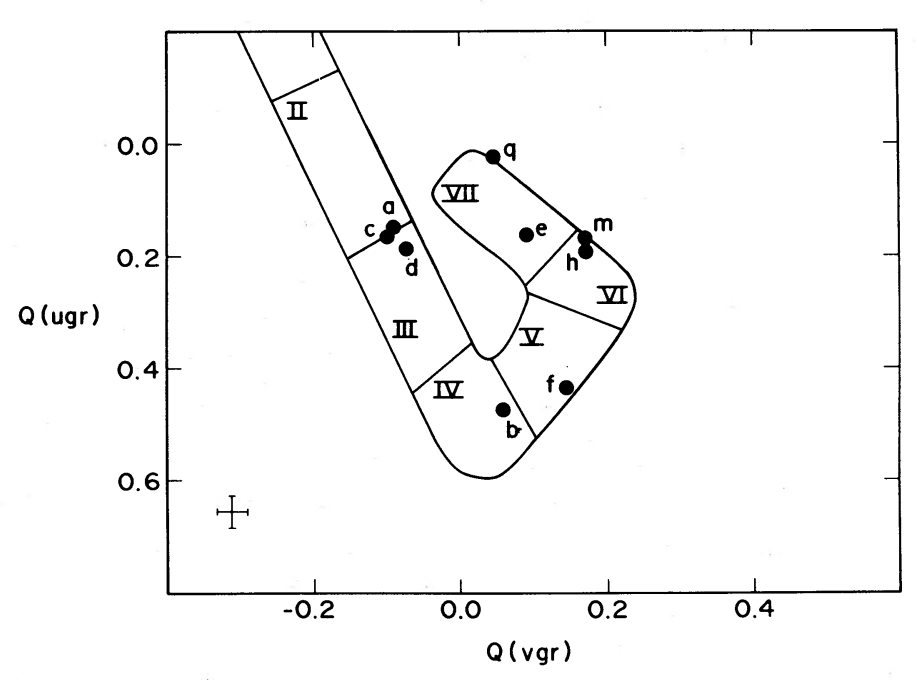

FIG. $2 b$

FIG. 2.- (a) M33 clusters in the $Q, Q$ diagram. The enclosed zone is that occupied by the Galaxy's globular clusters. The halo clusters of the Galaxy are confined to zone A. The sequence indicated is a theoretically predicted sequence for clusters of solar abundance; cluster ages in gyr are indicated along this sequence. (b) The same M33 clusters in the $Q, Q$ diagram. The enclosed zone is that occupied by clusters in the Magellanic Clouds. The subzones define the SWB classification scheme. 
M33 cluster $f$ has an age of about $3 \times 10^{9}$ years. Possibly clusters $b$ and $f$ are modestly metal poor, since the very similar clusters of the LMC such as NGC 1846 and NGC 2209 have abundances $[\mathrm{Fe} / \mathrm{H}]=-1$ according to Cohen (1982) and Rabin (1982). The Balmer lines dominate the spectra of these younger clusters (see Fig. 1), with those of cluster $f$ being somewhat weaker than the other young or intermediate-aged M33 clusters, but still clearly distinguished from those of truly old clusters.

\section{THE TRUE GLOBULARS OF M33}

Both spectra and photometry identify clusters e, h, q, and $\mathrm{m}$ as the only true analogs of galactic globular clusters among the M33 cluster sample. Based on Figure 1, they are ranked in order of increasing metallicity as $\mathrm{q}, \mathrm{e}, \mathrm{h}$, and $\mathrm{m}$, with $\mathrm{h}$ and $\mathrm{m}$ being essentially indistinguishable at $[\mathrm{Fe} / \mathrm{H}] \approx-1$ and clusters e and q significantly more metal poor. The spectrophotometric data of Figure 2 are completely consistent with this, differing only in inverting the metallicity of $m$ and $h$. SWB showed that for old globular clusters the parameters $Q(u g r)$ and $Q(v g r)$, which describe the integrated spectra, are closely correlated with the $S$ parameter of Searle and Zinn (1978), a reddening-free blanketing measure characteristic of the spectra of individual red giants in the clusters. Da Costa and Graham (1982) have used this fact to derive a relation between $[\mathrm{Fe} / \mathrm{H}]$ and the integrated-light $q$ parameters

$$
[\mathrm{Fe} / \mathrm{H}]=4.48 Q(\text { ugr })+3.12 Q(\text { vgr })-2.42 \pm 0.3 .
$$

This relation yields $[\mathrm{Fe} / \mathrm{H}]$ values of $-2.2,-1.4,-1.1$, and -1.0 for the clusters $\mathrm{q}, \mathrm{e}, \mathrm{m}$, and $\mathrm{h}$ respectively. Neither the mean nor the dispersion of these values differs significantly from the same quantities descriptive of the globular cluster systems of the LMC or of the Galactic halo.

The two type VI M33 clusters, Hiltner's clusters $h$ and m, have photometric properties that closely match those of the LMC clusters NGC 2121 and NGC 2155. It is not clear at present whether these clusters are younger than the more metal-poor type VII clusters or are simply the most metal-rich of a coeval globular cluster population. If clusters $h$ and $m$ are the same age as the Galaxy's globular clusters, then their abundances are close to $[\mathrm{Fe} / \mathrm{H}]=-1.0$, as we have already estimated. This is in excellent agreement with Cohen's (1982) determination of $[\mathrm{Fe} / \mathrm{H}]=-1$ for NGC 2121. Judging from their location in the $Q-Q$ plane, the clusters $\mathrm{h}$ and $\mathrm{m}$ are significantly less metal rich than 47 Tucanae.

Finally the two clusters $q$ and e that we have assigned to type VII have photometric properties rather similar to the LMC clusters NGC 1841 and NGC 2210, respectively, for which Cohen (1982) derives $[\mathrm{Fe} / \mathrm{H}]$ values of -2.3 and -1.7 . These values are in excellent agreement with the values -2.2 and -1.4 that we earlier derived for clusters $q$ and $e$ from Da Costa and Graham's relation. (Note that we do not have a high-quality spectrum for cluster q, and only an upper limit on its metallicity can be derived from Fig. 1.)

None of the M33 clusters occupies Zone B, the region of the $Q-Q$ plane characteristic of the more metal-rich of the Galaxy's globular clusters. Since only 20 of 84 galactic globular clusters in Zinn's (1980) compilation have $[\mathrm{Fe} / \mathrm{H}]>-1.0$, the absence of such a cluster from a sample of only four true globulars among the M33 cluster sample is not statistically significant. However, we have observed the reddest "probable clusters" not identified subsequently as background galaxies found by Christian and Schommer (1982).
Christian and Schommer (1982) stated that the sample of red clusters in M33 with photometry is now 13 objects, while the total number of such clusters in M33 predicted by Harris and Racine (1979) is 20. The luminosity function that Christian and Schommer derive for the red clusters resembles the "universal luminosity function" advocated for globular clusters by Harris and Racine (1979), but the M33 clusters are in the mean 0.6 mag too faint. That difference is removed by the new distance modulus of Sandage and Carlson (1983).

\section{CONSISTENCY OF THE INFRARED OBSERVATIONS WITH OPTICAL ANALYSES}

The $(J-K)_{0}$ and $(V-H)_{0}$ colors of eight M33 clusters are plotted in Figure 3. The median relationship for 40 M31 clusters is shown as the curved line. Also shown are all the LMC and SMC clusters measured by Persson et al. (1983) which have been classified by SWB. We have grouped the SWB classes, for the purposes of Figure 3, as follows: 1 stands for I; 2 for II and III; 5 for IV, V, and VI; and 7 for VII. (Note that the infrared photometric systems used to make the M33, M31, and LMC/SMC measurements have been compared using globular cluster stars in common and have been found indistinguishable).

Despite the rather large observational uncertainties in the M33 cluster data, it is clear that the infrared data corroborate the spectroscopic results discussed above. In particular:

1. Cluster a lies in a region of the diagram occupied by clusters in groups II and III (also V), and so is consistent with its location in the $Q-Q$ diagram as a II-III cluster.

2. Cluster $\mathrm{b}$ is quite red in both $(J-K)_{0}$ and $(V-K)_{0}$ and lies in a region occupied mostly by group IV, V, and VI objects (with one from group I). In Persson et al. (1983) objects with $(J-H)_{0}>0.6$ and $(H-K)_{0}>0.2$ were designated "IR

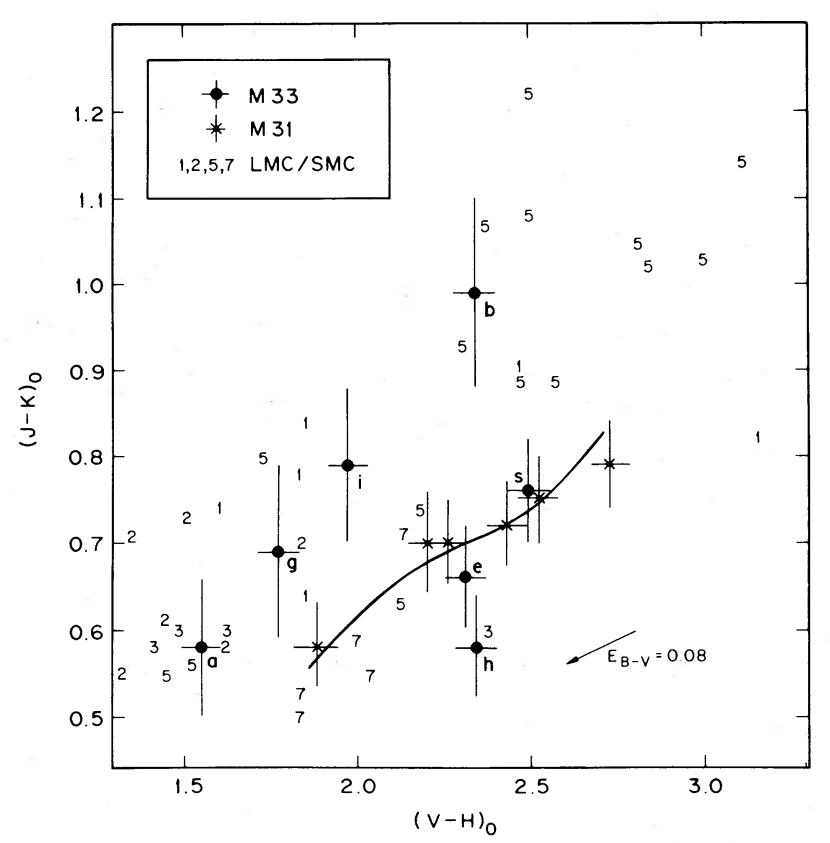

FIG. 3.- $(J-K)_{0}$ is plotted against $(V-H)_{0}$ for the clusters in M33 (solid circles) and in the Magellanic Clouds (numbers refer to SWB types; see text). The mean relationship for all globular clusters in M31 (Frogel, Persson, and Cohen 1980) is shown as a solid line. The individual M31 clusters used to transform the measured $r-H$ colors to $V-H$ are also shown. The error bars are $1 \sigma$. 
enhanced" and shown to contain carbon stars on the asymptotic giant branch. Although we lack $\mathrm{CO}$ data for cluster $\mathrm{b}$, it seems likely that it does indeed contain carbon stars, a finding which is not unexpected purely from its classification as a group IV cluster.

3. Clusters $\mathrm{e}, \mathrm{h}$, and $\mathrm{s}$ lie in the region of the diagram occupied by old metal-poor clusters, like those in M31. Cluster $\mathrm{h}$ was noted above to have a higher $[\mathrm{Fe} / \mathrm{H}]$ than cluster $\mathrm{e}$, whereas in Figure 3 they are not separated. Although we did not obtain a $J$ measurement for M33q, its $(V-H)_{0}$ color places it close to clusters e and $\mathrm{h}$, also consistent with its location in the $Q-Q$ plane.

We conclude from the infrared data that the energy distributions of the M33 clusters are consistent with their counterparts in the SWB classification of LMC, SMC and Milky Way clusters.

\section{a) Remarks on the Reddening Assumed}

We have adopted the value $E(B-V)=0.03 \pm 0.02$ of McClure and Racine (1969) for M33 which includes the foreground reddening only. For the true globulars we can use the $(\mathrm{V}-\mathrm{H})_{0}$ colors to estimate values of $[\mathrm{Fe} / \mathrm{H}]$ via the relationship for the $(V-K)_{0}$ colors of M31 clusters in Frogel, Persson, and Cohen (1980). On the Zinn scale $[\mathrm{Fe} / \mathrm{H}]_{\mathrm{Zinn}}=$ $2.33(V-K)_{0}-6.88$, and since $(V-K)_{0} \sim 8.5(H-K)_{0}+1.36$, we have

$$
[\mathrm{Fe} / \mathrm{H}]_{\mathrm{Zinn}}=2.64(\mathrm{~V}-\mathrm{H})_{0}-7.32 .
$$

We arrive at the estimates for the metallicities of the true globulars of $-1.2,-0.8,-1.1$, and -1.2 for clusters $\mathrm{e}, \mathrm{s}, \mathrm{h}$, and $\mathrm{q}$, respectively. These values are considerably higher than obtained from the spectroscopic data discussed earlier and suggest that our estimate for the reddening is too low, by $\sim 0.1$ in $E(B-V)$. Support for a generally larger value of $E(B-V)$ is found by plotting the (reddening-free) $Q$ values versus the raw $B-V$ colors for all the old globular clusters in M31 and M33. There is no systematic displacement of the M33 points in this plot, which thus rules out a difference in the reddenings of magnitude larger than $\Delta E(B-V)=0.02$. However, the foreground reddening for M31 (also determined by McClure and Racine 1969) is $0.11 \pm 0.02 \mathrm{mag}$. Based on these plots, cluster $q$ in particular may be somewhat more reddened than the other M33 globulars in our sample, perhaps being located behind the disk or a spiral arm of M33. This does not change the conclusions regarding the different stellar populations in clusters $\mathrm{a}, \mathrm{g}$, $\mathrm{i}$, and $\mathrm{b}$ compared to e, s, $\mathrm{q}$, and $\mathrm{h}$, as can be seen from the dereddening vector in Figure 3.

\section{b) The Carbon Star Contribution to the Light of the Intermediate Age Clusters}

We now compare cluster b to NGC 419 in the SMC. NGC 419 is known to contain at least eight carbon stars and have $K_{0}=7.5$ in a $60^{\prime \prime}$ diameter aperture (Persson et al. 1983). At the distance of M33 $\left[(m-M)_{0}=25.23\right]$ NGC 419 would appear as a cluster with $K_{0}=13.5$ in a 3.8 aperture, or 13.3 in a $5^{\prime \prime}$ aperture. This is about $1.5 \mathrm{mag}$ brighter than cluster $\mathrm{b}$ in M33 which has $K_{0}=14.8$ in a $5^{\prime \prime}$ aperture. If the carbon star production efficiency were the same in the two clusters, then we should expect two AGB carbon stars in cluster b. If, further, these two stars come from a luminosity function like that of carbon stars in the clusters and fields of the Magellanic Clouds, then they should contribute $K_{0}=15.8$ to the cluster light. This is quite consistent with the location of cluster $b$ in Figure 3 and with the result that on average about half the $K$ (or bolometric) light of intermediate age clusters in the Clouds is due to carbon stars (Persson et al. 1983). On the basis of only limited statistics, the carbon star production efficiency is thus comparable to that seen in intermediate age clusters in the Magellanic Clouds.

As pointed out by Christian and Schommer (1982), there are no known analogs of these clusters in our Galaxy of comparable luminosity. NGC 2158 , for example, is significantly fainter than even the mean of the LMC clusters of this age range.

\section{IMPLICATIONS AND SUMMARY}

Our major conclusion is that, on the basis of the limited statistics of the present sample, the luminous clusters of M33 have an age spread similar to those of the Magellanic Clouds and quite unlike those of the Galaxy or of M31. The three different observational techniques used here all give consistent age rankings for the young versus old M33 clusters; furthermore, the two reddening independent ones give the same metallicities for the four true globular clusters in the M33 sample. If the reddening normally used for M33 is underestimated by $\Delta E(B-V) \approx 0.1 \mathrm{mag}$, then the abundances of the four globular clusters in M33 inferred from broad-band colors also agree quantitatively with the values determined via reddening independent techniques.

The carbon star production rate in the intermediate age M33 clusters appears to be consistent with that of LMC clusters. One might therefore expect many field carbon stars to be found in M33.

As in the Magellanic Clouds, there appears to be an absence of M33 clusters from Zone B of Figure $2 a$. If this is not a result of our small sample, what does it imply? The figure shows that clusters of two types might be expected to inhabit this Zone: $(i)$ metal-rich clusters of the same age as the oldest clusters in the Galaxy, and (ii) clusters of roughly solar abundance and any age between 5 and 15 gyr. It is possible that the strong line globular clusters in the Galactic center contain representatives of both these types. It is interesting that van den Bergh (1969) pointed out that there is a range in hydrogen line strength among clusters of Morgan class VIII. Since such clusters do not contain horizontal-branch stars, this may be an indication of a range in cluster ages.

It is perhaps not too surprising that metal-rich old clusters failed to form in M33. What is more strange is the absence of old disk clusters. If clusters such as $\mathrm{M} 33 \mathrm{~b}$ and $\mathrm{f}$ were formed in M33's disk within the past $3 \mathrm{gyr}$, it is difficult to understand why one does not find twice as many formed in the era between 3 and 9 gyr ago. For a given initial cluster mass an older cluster will be fainter; clusters resembling $b$ and $f$ will be fainter by about 0.7 mag when they are 9 gyr old, according to the estimates of Searle, Sargent, and Bagnuolo (1973). It will present an interesting problem if deeper surveys of M33 cluster system fail to find these older disk clusters.

Emission-line objects in M33 (both H II regions and supernova remnants) are bright enough to permit detailed observational study. As reviewed by Pagel and Edmunds (1981), there is a strong radial abundance gradient, and the solar abundance is not reached until within $1^{\prime \prime}$ (less than $500 \mathrm{pc}$ ) of the center of the galaxy. The outer parts of the galaxy, where most of the clusters are found, have abundances between one-half and onetenth the solar value, comparable to that of the LMC or SMC gas. Thus the absence of metal-rich old disk clusters in both the Magellanic Clouds and M33 may not be entirely unexpected. 
The abundance range of the four true globulars of M33 is comparable to that found by Cohen (1982) for the equivalent group of clusters in the LMC.

The strong similarity of the cluster system of M33 with those of the Magellanic Clouds and the contrast with those of the Galaxy or M31 suggest that gas-rich late-type systems may have a star formation history quite different from that of earlier type galaxies.

\section{REFERENCES}

Aaronson, M., and Mould, J. R. 1982, Ap. J. Suppl., 48, 161.

Bagnuolo, W. G. 1976, Ph.D. thesis, California Institute of Technology.

Christian, C. A., and Schommer, R. A. 1982, Ap. J. Suppl., 49, 405.

. 1983, in Internal Kinematics and Dynamics of Galaxies, ed. E. Athanassoula (D. Reidel), p. 365.

Cohen, J. G. 1982, Ap.J., 258, 143.

$$
\text { .1983, Ap. J., 270, } 654 .
$$

DaCosta, G. S., and Graham, J. A. 1982, Ap. J., 261, 70

Danziger, I. J. 1973, Ap. J., 181, 641 .

Elias, J. H., Frogel, J. A., Matthews, K., and Neugebauer, G. 1982, A.J., 87, 1029.

Frogel, J. A., Cohen, J. G., and Persson, S. E. 1983, Ap. J., 275, 771.

Frogel, J. A., Persson, S. E., and Cohen, J. G. 1980, Ap. J., 240, 785 (FPC 80)

Harris, W. E., and Racine, R. 1979, Ann. Rev. Astr. Ap., 17, 241.

Hiltner, W. 1960, Ap. J., 131, 163.

Hodge, P. 1981, in Astrophysical Parameters for Globular Clusters, ed. A. G.

Davis Philip and D. S. Hayes (Schenectady: L. Davis Press), p. 205.

Humphreys, R. M., and Sandage, A. R. 1980, Ap. J. Suppl., 44, 319.

Kaufman, M. 1981, Ap. J., 250, 534.

McClure, R. D., and Racine, R. 1969, A.J., 74, 1000.

Melnick, J., and D'Odorico, S. 1978, Astr. Ap. Suppl., 34, 349.

Oke, J. B., and Gunn, J. E. 1982, Pub. A.S.P., 94, 586.

Oke, J. B., and Schild, R. 1970, Ap. J., 161, 1015.

Pagel, B. E. J., and Edmunds, M. G. 1981, Ann. Rev. Astr. Ap., 19, 77.

Persson, S. E., Aaronson, M., Cohen, J. G., Frogel, J. A., and Matthews, K. 1983, Ap.J., 266, 105.

Persson, S. E., Cohen, J. G., Sellgren, K., Mould, J., and Frogel, J. A. 1980, Ap. $J ., 240,779$.

Rabin, D. 1982, Ap. J., 261, 85 .

Rogstad, D. H., Wright, M. H. C., and Lockhart, I. A. 1976, Ap. J., 204, 703.

Sandage, A. R., and Carlson, G. 1983, Ap. J. (Letters), 267, L25.

Sandage, A. R., and Tammann, G. A. 1981, A Revised Shapley-Ames Catalog of Bright Galaxies (Carnegie Institution of Washington Publication 635).

Searle, L., Sargent, W. L. W., and Bagnuolo, W. G. 1973, Ap. J., 179, 427.

Searle, L., Wilkinson, A., and Bagnuolo, W. G. 1980, Ap. J., 239, 803 (SWB).

Searle, L., and Zinn, R. 1978, Ap.J., 225, 357.

Thuan, T. X., and Gunn, J. E. 1976, Pub. A.S.P., 88, 543.

van den Bergh, S. 1969, Ap. J. Suppl., 19, 145.

Zinn, R. 1980, Ap. J. Suppl., 42, 19.

J. G. COHEN : California Institute of Technology, 105-24, Pasadena, CA 91125

S. E. Persson and L. Searle: Mount Wilson and Las Campanas Observatories, 813 Santa Barbara Street, Pasadena, CA 91101 